\title{
Gamma Evaluation with Octavius 4D Phantom for Pre- Treatment of Modern Radiotherapy Treatment Techniques
}

\author{
Srivastava $\mathbf{R P}^{* 1,2}$, Basta $\mathrm{K}^{2}$, Thevissen $\mathrm{K}^{2}$, Junius $\mathrm{S}^{2}$, Vandeputte $\mathrm{K}^{2}$ \\ and Wagter $\mathrm{CD}^{1}$ \\ ${ }^{1}$ Department of Radiation Oncology, Ghent University Hospital, Belgium \\ ${ }^{2}$ Radiotherapy Association Meuse Picardie, Centre Hospitalier Mouscron, Belgium
}

\section{Research Article}

Volume 2 Issue 2

Received Date: November 22, 2019

Published Date: December 09, 2019

*Corresponding author: Srivastava RP, Radiotherapy Association Meuse Picardie, Centre Hospitalier Mouscron, Avenue de Fécamp 49, 7700 Mouscron, Belgium, Tel: +3256858363; Email: rajupsrivastava@hotmail.com

\section{Abstract}

Purpose: In modern radiotherapy techniques such as intensity-modulated radiation therapy (IMRT) and volume modulated arc therapy (VMAT), the quality assurance (QA) process is vital. The goal of the study was to verify the treatment planning dose delivered during delivery of complex treatment plans. The QA standard is to perform patientspecific comparisons between planned doses and doses measured in a phantom.

Materials and Methods: Ninety-five complex IMRT and VMAT plans for different pathologies planned using Eclipse treatment planning system (TPS). The Octavius 4D phantom has been used to verify patient specific quality assurance of all VMAT plans calculating with different algorithms.

Conclusion: Overall, good agreement was observed between measured and calculated doses in most cases with gamma values above 1 in $>95 \%$ of measured points in volumetric 3D analysis and 1 in $>90 \%$ in $2 \mathrm{D}$ analysis. The Octavius $4 \mathrm{D}$ phantom is an effective and efficient method for patient specific QA.

Keywords: IMRT; VMAT; Quality Assurance; Gamma Value; Treatment Planning System

Abbreviations: IMRT: Intensity-Modulated Radiation Therapy; MLC: Multi-Leaf Collimator; MU: Monitor Unit; TPS: Treatment Planning Systems; PTV: Planning Target Volume; FiF: Field in Filed; AAA: Anisotropic Analytical Algorithm; PDD: Percentage Depth Dose.

\section{Introduction}

Intensity-modulated radiation therapy (IMRT) and volumetric modulated arc therapy VMAT) can deliver optimal dose distributions delivering prescription doses to the target volumes, which are enough to control tumor cells, while reducing doses to normal tissues by modulating photon beam intensities [1-3]. IMRT modulation photon beam intensities by varying the multileaf collimator (MLC) positions.

VMAT modulating the multi-leaf collimator (MLC) positions, gantry rotation speed, and dose rates has been broadly adopted in the clinic, having benefits of delivering prescription dose to target volume while sparing normal 


\section{International Journal of Nuclear Medicine \& Radioactive Substances}

tissue. It has shown that VMAT can achieve a similar plan quality and monitor unit (MU) effectiveness as compared to intensity modulated radiation therapy (IMRT) resulting in shortening the treatment time in the clinic [4]. Intensity-modulated radiation therapy (IMRT) and VMAT can produce highly conformal radiation dose distributions and enhance treatment localization. These complex treatment techniques also place higher demands on dose calculation algorithms in terms of both accuracy and computation speed $[5,6]$. With the increasing popularity of IMRT and VMAT techniques in clinics, accuracy in treatment planning systems (TPSs) has always been a concern in modern radiotherapy.

Dose calculation accuracy is one of the most important steps in the radiation therapy treatment process, however, the dose calculation process is imperfect due to measurement uncertainties, inadequacies in beam modeling, and inherent limitations in the algorithms.

The planning process of IMRT and VMAT are not intuitive, which is an inverse or forward planning with optimization algorithms, and the delivery mechanisms of IMRT and VMAT are complex involving various mechanical parameters [7,8]. Moreover, calculations of the small or irregular fields which are frequently used for both IMRT and VMAT are not accurate even with the state-of-the-art dose calculation algorithms [9,10]. Therefore, there is potential for both IMRT and VMAT to cause differences between the calculated dose distribution and the actually delivered dose distribution to a patient [3]. This can result in unintended treatment which is detrimental to patients, which should be avoided. In this respect, patient-specific quality assurance QA) for every patient treated with IMRT or VMAT technique is performed in the clinic before patient treatment [11-13].

In this study, the pre-treatment patient-specific quality assurance for these VMAT cases were completed using the OCTAVIUS phantom combined with Detector 1500 array T10044 (PT W-Freiburg, Freiburg, Germany). We analyzed the gamma passing rates in $2 \mathrm{D}$ and $3 \mathrm{D}$ acquired with Octavius phantom for various pathology for both IMRT and VMAT.

\section{Materials and Methods}

Measurements were performed with Varian Clinac iX accelerators equipped with MLC with a Millennium 120 multileaf collimator (Varian Medical Systems, Palo Alto, CA, USA) using 6 and 18 MV photon beams.

\section{Patients Selection}

Ninety-five patients were included for this study who underwent treatment with VMAT technique in our institute between February-June 2019. The pathologies were 20 patients for each for prostate cancer, head and neck, Breast and 15 patients for lung. The lung cancers were treated with stereotactic body radiation therapy (SBRT) technique.

\section{Dose Calculation and Planning}

All three cases of VMAT plans of patients were generated in the Eclipse TPS for a Clinac iX equipped with a 120 Millennium MLC. The VMAT plan was created in Acuros AXBm. For prostate cases, the total dose prescribed to the planning target volume (PTV) was 76 Gy with a daily dose of 2.0 Gy in 38 fractions. The prostate VMAT plans were generated using two-full-arcs with 6 \& $18 \mathrm{MV}$ photon beams as needed. The head and neck VMAT plans were generated by using a two or three-full-arcs with $6 \mathrm{MV}$ photon beam and the total prescription dose to PTV was $70 \mathrm{~Gy}$ (2.0 Gy/fraction). The prostate and head \& neck cases were treated in simultaneous integrated boost (SIB) fractionation schemes. The greatest dose was mentioned for the study. The breast cases were treated in field in filed (FiF) techniques and the dose to PTV (40.05 Gy) was given in 15 fractions. The dose to CTV was $10 \mathrm{~Gy}$ (2Gy/fraction). For lung SBRT cases, prescription dose to PTV was 48 Gy in 4 fractions of $80 \%$ of isodose. The VMAT plans for lung SBRT were made using two-partialarcs with $6 \mathrm{MV}$ photon beam. The dose calculation grid used in this study was $2.5 \mathrm{~mm}$ except for $1.0 \mathrm{~mm}$ for lung SBRT cases. All the cases were planned by algorithm Anisotropic Analytical Algorithm (AAA), Acuros XB (AXB). AXB provides two dose reporting modes: dose-to-water $(\mathrm{AXBw})$ and dose-to-medium (AXBm).

\section{Octavius Phantom}

The 2D-Array together with Octavius $®$-4D (PTWFreiburg, Freiburg, Germany) are widely described in the literature [14]. Briefly, the 2D-Array 1500 consists of 1405 vented ionization chambers, each having an entrance area of $4.4 \times 4.4 \mathrm{~mm} 2$ and a height of $3 \mathrm{~mm}$, resulting in an ionization volume of $0.058 \mathrm{~cm} 3$.The chambers are supplied with a voltage of $1000 \mathrm{~V}$. They are arranged in rows, and the center-to-center distance between the chambers in each row is $10 \mathrm{~mm}$. The distance between the rows is $5 \mathrm{~mm}$. A checkerboard pattern of the chamber arrangement has been achieved by an offset of each second row of chambers, compared 
with its neighbour rows, of $5 \mathrm{~mm}$ in the row direction. Thereby, the nearest neighbour distance between chambers, measured along the diagonals, is $7.1 \mathrm{~mm}$. This arrangement of the chambers results in a spatial sampling frequency of $0.1 \mathrm{~mm}-1$ in each row or column and 0.14 $\mathrm{mm}-1$ in the diagonal direction. By merging two measurements with the array shifted by $5 \mathrm{~mm}$ in lateral or longitudinal direction, the sampling frequency along each row and column can be doubled to $0.2 \mathrm{~mm}-1$. The manufacturer-specified reference point is $7.5 \mathrm{~mm}$ beneath the surface and marked at the outer wall of the array.

The 2D-Array 1500 is inserted into a motorized cylindrical polystyrene phantom, (diameter and length 32 $\mathrm{cm}$ and $34.3 \mathrm{~cm}$ respectively). An inclinometer mounted on the gantry ensures that the rotation unit always rotates along with the gantry, thus keeping the 2D array perpendicular to the beam axis always. The inclinometer is connected via wifi to a control unit as shown in fig 1 that transfers the movement information to the phantom and acquires dosimetric data every $200 \mathrm{~ms}$. The beam always hits the detector array in a perpendicular way because the same face of the detector follows the gantry, so no correction factors are required (Figure 1).

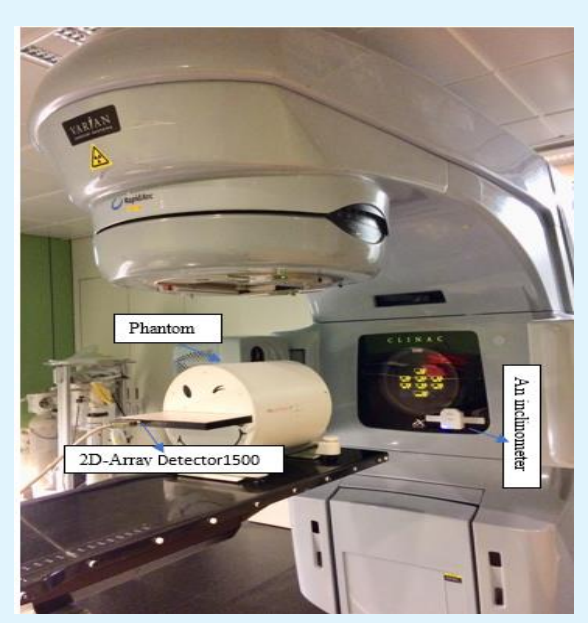

Figure 1: Standard set up of Octavius 4D system in measurement position with Detector 1500 inserted in a cylindrical phantom that rotates synchronously with gantry and the inclinometer attached to gantry.

Upon loading this file in the Verisoft software, the 3D dose in a homogeneous cylindrical phantom is reconstructed: for every stored gantry angle, the 2D measurement data are extrapolated to the rest of the cylinder by applying a percentage depth dose (PDD) curve through every measurement point. The total 3D dose is then reconstructed as the sum of these individual contributions and linearly interpolated to a user specified dose grid.

To evaluate the dosimetric agreement between measured and calculated dose, we make use of the gamma evaluation method implemented in the Verisoft 7.2.0 version. This calculation of the gamma index is based on the concept of Low, et al. [15].

\section{Results}

All plans were analyzed using the three parameters, the \%DA (limit 3\%), DTA (limit $3 \mathrm{~mm}$ ), and g-pass rate with the $3 \%$ dose tolerance and $3 \mathrm{~mm}$ distance to agreement in relation to the treatment planning system (Table 1).

The 3D dose reconstruction in the Octavius 4D system allows the user to select any plane (axial, coronal and sagittal) for the evaluation. With the use of Octavius 4D system, it is possible to compare and analyze volumetric dose field by field or composite field for VMAT in a single measurement, thus the user gains confidence about the delivery of even complex radiotherapy plans. Potential improvements in the device could be achieved with closer detector spacing for better resolution.

Figure 2 shows the result of 3 beams SBRT dose map in eclipse, calculated dose matrices in Octavius 4D phantom and gamma analysis of lung case in transversal, sagittal and coronal plane respectively. It should be noted that for all plans, our clinical standard of $90 \%$ or greater for the gamma index percentage was achieved.

All these cases were also studied in portal dosimetry and compared to Octavius 4D. These results are not presented here Sharma, et al. [16]. Validated the portal dosimetry for IMRT in comparison with the results using 2D ion chamber array with 14 IMRT cases. They concluded both 2D ion chamber array and the portal dosimetry showed comparable results, therefore, both can be used for the patient-specific QA of IMRT Fogliata, et al. [17] validated the VMAT technique with portal dosimetry by utilizing a total of 275 patient cases. 


\section{International Journal of Nuclear Medicine \& Radioactive Substances}

\begin{tabular}{|c|c|c|c|c|}
\hline \multirow{4}{*}{ Pathologies } & \multicolumn{4}{|c|}{ Gamma passing rates (\%) for AAA } \\
\hline & \multicolumn{3}{|c|}{ Planar analysis } & \multirow{3}{*}{$\begin{array}{l}\text { Volumetric 3D } \\
\text { analysis }\end{array}$} \\
\hline & \multicolumn{3}{|c|}{ 2D } & \\
\hline & Coronal & Sagittal & Transversal & \\
\hline Prostate & $95,4 \pm 1,2$ & $96,2 \pm 0,2$ & $96,8 \pm 0,5$ & $97,9 \pm 1,1$ \\
\hline Head \& neck & $97,2 \pm 0,8$ & $97,6 \pm 1,3$ & $98,1 \pm 0,7$ & $98,1 \pm 1,2$ \\
\hline Lung & $91,8 \pm 1,9$ & $91 \pm 0,5$ & $94,3 \pm 1,3$ & $97,4 \pm 1,6$ \\
\hline Breast & $95,2 \pm 0,8$ & $97,1 \pm 1,3$ & $96.8 \pm 0,7$ & $98,7 \pm 1,3$ \\
\hline \multirow{4}{*}{ Pathologies } & \multirow{2}{*}{\multicolumn{3}{|c|}{$\begin{array}{l}\text { Gamma passing rates }(\%) \text { for } \mathbf{A X B}_{\mathrm{w}} \\
\text { Planar analysis }\end{array}$}} & \\
\hline & & & & \\
\hline & \multicolumn{3}{|c|}{$2 \mathrm{D}$} & Volumetric 3D analysis \\
\hline & Coronal & Sagittal & Transversal & \\
\hline Prostate & $95,6 \pm 0,4$ & $95,8 \pm 1,2$ & $96,2 \pm 0,4$ & $97,5 \pm 0,5$ \\
\hline Head \& neck & $96,7 \pm 0,3$ & $96,9 \pm 0,3$ & $98,2 \pm 0,6$ & $98,7 \pm 0,3$ \\
\hline Lung & $99,1 \pm 0,2$ & $99,2 \pm 0,3$ & $99,6 \pm 0,1$ & $99,7 \pm 0,2$ \\
\hline Breast & $95,2 \pm 0,5$ & $96,1 \pm 1,1$ & $96.0 \pm 0,3$ & $98,2 \pm 1,0$ \\
\hline \multirow{4}{*}{ Pathologies } & \multicolumn{4}{|c|}{ Gamma passing rates $(\%)$ for $\mathrm{AXB}_{\mathrm{m}}$} \\
\hline & \multicolumn{3}{|c|}{ Planar analysis } & \multirow{3}{*}{ Volumetric 3D analysis } \\
\hline & \multicolumn{3}{|c|}{$2 \mathrm{D}$} & \\
\hline & Coronal & Sagittal & Transversal & \\
\hline Prostate & $96,1 \pm 1,1$ & $96,2 \pm 1,2$ & $97,4 \pm 0,5$ & $99,4 \pm 0,5$ \\
\hline Head \& neck & $97,3 \pm 1,7$ & $97,8 \pm 1,0$ & $97,6 \pm 1,1$ & $99,6 \pm 0,2$ \\
\hline Lung & $94,8 \pm 1,2$ & $94,1 \pm 1,3$ & $99,6 \pm 0,1$ & $99,7 \pm 0,2$ \\
\hline Breast & $96,1 \pm 0,8$ & $96,1 \pm 1,1$ & $96.7 \pm 1,3$ & $99,2 \pm 0,5$ \\
\hline
\end{tabular}

Table 1: summarizes the results of average g-passing rate of different pathologies in our center in 2D and 3D analysis for $\mathrm{AAA}, \mathrm{AXBw}$ and AXBm. The gamma pass rates over all plans were higher than $\leq 97.0 \%$ volumetric $3 \mathrm{D}$ analysis.

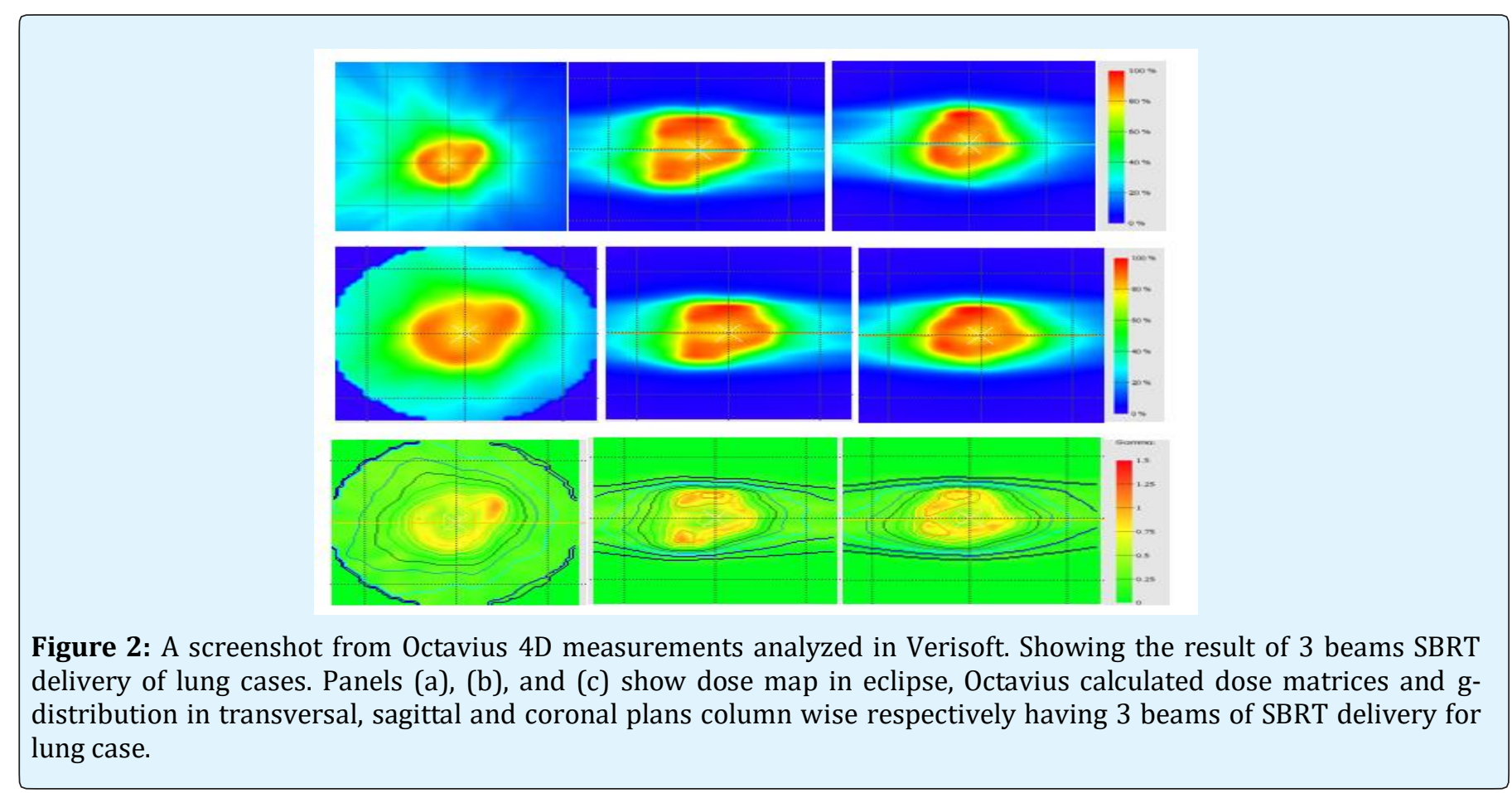




\section{International Journal of Nuclear Medicine \& Radioactive Substances}

\section{Discussion}

Results underline that several factors affect plan evaluation when using Octavius ${ }^{\circledR}-4 \mathrm{D}$, and they are especially enhanced if the more restrictive local $\gamma$-index computation approach is used. Indeed, the global $\gamma$-index produces more homogeneous results with higher passing rates because its tolerance level is computed with respect to the value of maximum dose. The 2D approach considers each slice as independent of the surrounding volume, with the drawback that results are strongly dependent on the chosen plane, without a certain significant correlation between the magnitude of errors of different plans [18]. The 3D analysis allows a slice-by-slice evaluation taking into account also the neighboring slices. Our results confirmed that the single slice evaluation (2D) had always a worse agreement compared to 3D and volumetric $\gamma$-index. Pulliam [18] compared the two gamma results using a Monte Carlo computation as reference dose distribution and quantified the increase of passing pixels percent up to $3.2 \%$ in the $3 \mathrm{D}$ analysis, confirming our findings.

\section{Conclusion}

The results indicate that Octavius $4 \mathrm{D}$ phantom is an effective and efficient method for patient specific QA. This system is accurate dosimetrically and measurement control and three-dimensional analysis that are highly adapted to the complexities of modulated irradiation. The Octavius $4 \mathrm{D}$ phantom is a suitable tool in the dosimetric verification of the VMAT and has proven to be fast and reliable for patient specific QA.

\section{References}

1. Zhang P, Happersett L, Hunt M, Jackson A, Zelefsky M, et al. (2010) Volumetric modulated arc therapy: planning and evaluation for prostate cancer cases. International Journal of Radiation Oncology Biology Physics 76(5): 1456-1462.

2. Studenski MT, Bar Ad V, Siglin J, Cognetti D, Curry J, Tuluc M, et al. (2013) Clinical experience transitioning from IMRT to VMAT for head and neck cancer. Medical Dosimetry 38(2): 171-175.

3. Ezzell GA, Burmeister JW, Dogan N, LoSasso TJ, Mechalakos JG, et al. (2009) IMRT commissioning: multiple institution planning and dosimetry comparisons, a report from AAPM Task Group 119. Medical Physics 36 (11): 5359-5373.
4. Delivery accuracy of VMAT, The British Journal of Radiology. 88: 20140698.

5. Van Esch A, Tillikainen L, Pyykkonen J, Tenhunen M, Helminen $\mathrm{H}$, et al. (2006) Testing of the analytical anisotropic algorithm for photon dose calculation. Medical Physics 33(11): 4130-4148.

6. Ding GX, Duggan DM, Coffey CW (2007) Comm ent on Testing of the analytical anisotropic algorithm for photon dose calculation. Medical Physics 34(8): 3414.

7. Brahme A (1988) Optimization of stationary and moving beam radiation therapy techniques. Radiotherapy and Oncology 12(2): 129-140.

8. Otto K (2008) Volumetric modulated arc therapy: IMRT in a single gantry arc. Medical Physics 35(1): 310-317.

9. Park JM, Park SY, Kim H (2015) Modulation index for VMAT considering both mechanical and dose calculation uncertainties. Physics in Medicine \& Biology 60(18): 7101-7125.

10. Park SY, Kim IH, Ye SJ, Carlson J, Park JM (2014) Texture analysis on the fluence map to evaluate the degree of modulation for volumetric modulated arc therapy. Medical Physics 41(11): 111718.

11. Fredh A, Scherman JB, Fog LS, Rosenschold PM(2013) Patient QA systems for rotational radiation therapy: a comparative experimental study with intentional errors. Medical Physics 40(3): 031716.

12. Heilemann G, Poppe B, Laub W (2013) On the sensitivity of common gamma-index evaluation methods to MLC misalignments in Rapidarc quality assurance. Medical Physics 40(3): 031702.

13. Kim J, Park SY, Kim HJ, Kim JH, Ye SJ, et al. (2014) The sensitivity of gamma-index method to the positioning errors of high-definition MLC in patient-specific VMAT QA for SBRT. Radiation Oncology 9: 167.

14. Stathakis S, Myers P, Esquivel C, Mavroidis P, Papanikolaou N (2013) Characterization of a novel 2D array dosimeter for patient-specific quality assurance with volumetric arc therapy. Medical Physics 40(7): 071731. 
15. Low DA, Harms WB, Mutic S, Purdy JA (1998). A technique for the quantitative evaluation of dose distributions. Medical Physics 25(5): 656-661.

16. Sharma DS, Mhatre V, Heigrujam M, Talapatra K, Mallik S (2010) Portal dosimetry for pretreatment verification of IMRT plan: a comparison with 2D ion chamber array. Journal of Applied Clinical Medical Physics 11(4): 3268.
17. Fogliata A, Clivio A, Fenoglietto P, Hrbacek J, Kloeck S, et al. (2011) Quality assurance of RapidArc in clinical practice using portal dosimetry. The British Journal of Radiology 84(1002): 534-545.

18. Pulliam KB, Huang JY, Howell RM, Followill D, Bosca $\mathrm{R}$, et al. (2014) Comparison of $2 \mathrm{D}$ and $3 \mathrm{D}$ gamma analyses. Medical Physics 41(2): 021710. 